\title{
Sistema de Aprendizaje Basado en la Investigación aplicado en la Unidad de Aprendizaje Salud Pública en el CICS UMA IPN
}

\section{Research-Based Learning System applied in the Public Health Learning Unit at CICS UMA IPN}

DOI: 10.46932/sfjdv2n5-098

Received in: Oct 1st, 2021

Accepted in: Dec 30th, 2021

\author{
Blanca Elisa Pérez Magaña \\ $\mathrm{M}$ en $\mathrm{C}$ \\ Instituto Politécnico Nacional \\ E-mail: blancaelisa1@hotmail.com \\ Claudio Francisco Hernández Rodríguez \\ $\mathrm{M}$ en $\mathrm{C}$ \\ Instituto Politécnico Nacional \\ E-mail: chilapense@gmail.com \\ Carlos Quiroz Téllez \\ M C B \\ Instituto Politécnico Nacional \\ E-mail: cquirozte@ipn.mx
}

\begin{abstract}
RESUMEN
Los Modelos Educativo Institucional y del CICS UMA han servido de plataforma para la formación de profesionales con capacidad de generación de conocimientos a través de la aplicación de protocolos de investigación para lograr los objetivos específicos de las Unidades de Aprendizaje, con lo que la construcción de estructuras de conocimientos profesionales se fundamenta en un proceso constructivo mediante la aplicación de habilidades cognitivas. Así, en el desarrollo de la Unidad de Aprendizaje Salud Pública se aplicó el Sistema de Aprendizaje Basado en la Investigación, SABI, para poder alcanzar los objetivos: General de la Unidad de Aprendizaje y específicos para cada Unidad temática Esta estrategia permitió que los alumnos pudiesen contar con el primer elementos de construcción de sus estructuras cognitivas en materia de Salud Pública, mediante la elaboración de lecturas críticas para el desarrollo de los contenidos, mismo que sirve de materia prima para la construcción de marco teórico conceptual del proyecto de investigación. Con el trabajo de campo el alumno compara la realidad con la teoría y obtiene nuevos conocimientos, mismo que pone a disposición de la comunidad, con lo que cumple con su función social, que exige la formación profesional en Salud Pública. Mediante distintas plataformas que ofrece la WEB, dichos conocimientos se presentaron a la comunidad a través de videos sobre promoción de la salud visual para jóvenes que estudian nivel superior en México y hacen uso de los equipos de comunicación, tales como: teléfonos inteligentes, laptop, tablet, videojuegos.
\end{abstract}

Palabras clave: SABI, síndrome computador, salud visual.

\section{ABSTRACT}

The Institutional Educational Models and the CICS UMA have served as a platform for the training of professionals with the capacity to generate knowledge through the application of research protocols to 
achieve the specific objectives of the Learning Units, with which the construction of structures professional knowledge is based on a constructive process through the application of cognitive skills. Thus, in the development of the Public Health Learning Unit, the Research-Based Learning System, SABI, was applied in order to achieve the objectives: General of the Learning Unit and specific for each thematic unit.This strategy allowed the students to be able to have the first elements of construction of their cognitive structures in Public Health, through the preparation of critical readings for the development of the contents, which serves as raw material for the construction of the conceptual theoretical framework of the research project. With fieldwork, the student compares reality with theory and obtains new knowledge, which he makes available to the community, thereby fulfilling his social function, which requires professional training in Public Health. Through different platforms offered by the WEB, said knowledge was presented to the community through videos on promoting visual health for young people who study higher level in Mexico and make use of communication equipment, such as: smartphones, laptop, tablet, video games.

Keywords: SABI, computer syndrome, visual health.

\section{INTRODUCCIÓN}

El Centro Interdisciplinario de Ciencias de la Salud (CICS, UMA), al ser dependiente del Instituto Politécnico Nacional, tiene como guía de trabajo académico cotidiano las estrategias educativas que emanan de los elementos del Modelo Educativo del Instituto, garantizando así un perfil de nuestros egresados con características de calidad comunes para todos los profesionales formados en el Instituto, basadas en la equidad, pertinencia, relevancia, eficiencia y eficacia; logrado mediante procesos educativos caracterizados por una mayor correspondencia entre los contenidos y los resultados del quehacer académico, con las necesidades y expectativas de la sociedad y de los estudiantes.

Los elementos del Modelo Institucional son:

- Centrado en el aprendizaje, el estudiante es el centro de atención del proceso académico, considerándolo un individuo que construye su propio conocimiento con el apoyo del profesor.

- Una formación integral y de alta calidad científica, tecnológica y humanística, y que combine equilibradamente el desarrollo de conocimientos, actitudes, habilidades y valores.

- Que desarrolle facultades para una formación sólida y facilite el aprendizaje autónomo.

- Procesos educativos flexibles, innovadores que le permitan su relación con el entorno.

- Capaces de combinar la teoría y la práctica y contribuyan al desarrollo sustentable de la nación. ${ }^{1}$

Por otro lado, el CICS UMA tiene una trayectoria propia con peculiaridades que emanan de los principios que le dieron origen y que están expresados en el objeto de estudio del Centro: El hombre y el proceso salud-enfermedad. Para ello se conjugan estrategias enfocadas hacia el mejoramiento de la salud de nuestro país, mediante el trabajo interdisciplinario y con la participación de comunidad.

La metodología del CICS UMA está enfocada al estudio de los problemas que afectan a la sociedad visualizados no solo como un efecto provocado por los agentes patógenos sino por influencias 
ajenas a dichos agentes, esto es considerando los determinantes sociales que emanan del proceso mismo del desarrollo social, económico y político del país.

\section{MARCO TEÓRICO}

La filosofía del CICS UMA se ve reflejada en las siguientes políticas que rigen el quehacer académico y de integración social que lleva a cabo:

1. Integración práctica-teoría-práctica.

La relación permanente del alumno como sujeto cognoscente, con la teoría y la práctica integrada, evoluciona de tal manera que habiendo sido resuelto un problema elemental, el conocimiento continuará avanzando a grados mayores de complejidad aportando nuevos esquemas de atención y resolución a los problemas de salud de la comunidad.

\section{Estructura interdisciplinaria.}

El CICS tiene como propósito formar profesionales que aborden la salud del hombre de manera integral, lo cual obliga no solo a aplicar conocimientos de una sola disciplina o área de conocimientos, realizarlo de esta manera, la atención sería incompleta, por ello la necesidad de la interdisciplinaridad; la formación de equipos de salud interdisciplinarios para la atención de la salud permite abordar una realidad y a partir de ahí instrumentar técnicas y estrategias comunes y específicas de cada profesión, brindando una atención integral al individuo, familia y comunidad.

3. Desarrollo de la comunidad y compromiso social.

El desarrollo comunitario es concebido a través de diferentes niveles de participación y con acciones específicas a desarrollar, donde a cada uno de los entes participantes le corresponderá la puesta en marcha de la parte del plan que se le asigna, para la transformación social en beneficio de la salud de la comunidad. Se cumple con ello con uno de los elementos promovidos por la Organización Mundial de la Salud, OMS: Empoderamiento social.

4. Utilización óptima de recursos humanos y materiales.

Destaca en esta política: la utilidad del conocimiento para la sociedad y la contribución del CICS en la elevación del nivel de salud en la medida que la comunidad participe en las tareas de salud.

5. Integración docente-asistencial.

Es en la práctica social donde se realiza la integración docencia-servicio, mediante actividades que los alumnos desarrollan y realizan con el propósito de lograr una transformación de la realidad donde las llevan a cabo y una integración de los aspectos teóricos obtenidos en el aula en su vinculación con determinados tipos de práctica.

6. Servicio social continuo.

Desde su ingreso la formación profesional del alumno se desarrolla en las comunidades del área de influencia del Centro, en el aula, en las instalaciones de la misma institución y en las instalaciones del sistema regionalizado de salud; de esta manera el alumno desarrolla una retroalimentación constante de su responsabilidad social como estudiante y como futuro profesional egresado del CICS UMA. ${ }^{2}$ 
Por otro lado, el ejercicio docente obliga a la formación y desarrollo continuo del profesor, estableciendo como prioridades la mejora continua de su práctica docente y de investigación. Producto de lo anterior fue la conceptualización de un sistema de aprendizaje basado en la investigación científica, presentado ya en otros espacios, y al que se le ha identificado como: Sistema de Aprendizaje Basado en la Investigación, $\mathrm{SABI}{ }^{3}$.

En el contexto que plantea el Modelo Educativo Institucional y las políticas, que materializan la filosofía del CICS, y con el planteamiento que se hace en el Sistema de Aprendizaje Basado en la Investigación, las preguntas que nos planteamos ahora son: ¿Se alcanzan con este sistema de aprendizaje los objetivos del Modelo Educativo Institucional y se ajusta el resultado del aprendizaje a las Políticas del CICS, y con ello se forman los profesionales en el área de ciencias de la salud que la sociedad demanda? ¿Cómo se puede operar y llevar a la práctica en el aula el SABI, logrando con ello que durante el propio aprendizaje los alumnos vayan adquiriendo no solo las aptitudes sino también las actitudes profesionales en beneficio de la sociedad a la que están llamados a servir? ¿El CICS cumple con su responsabilidad social como formadores de profesionales en ciencias de la salud?

Ante estos planteamientos nos planteamos algunas estrategias educativas que tienen como principio lo que es el fundamento del SABI:

- Acuerdos con los alumnos para llevar a cabo la operación de las unidades con la participación diaria de ellos mediante lecturas críticas que den respuesta a los planteamientos de los contenidos de la Unidad de Aprendizaje

- Establecimiento de un proyecto final que de rumbo al aprendizaje al operar la Unidad de Aprendizaje.

- Especificar los resultados del aprendizaje que beneficiarán no solo el aprendizaje y formación del estudiante sino también su respuesta profesional, durante su propio proceso formativo, a las necesidades de la sociedad.

- Determinar las responsabilidades tanto del profesor como del alumno para llevar a cabo el proceso enseñanza aprendizaje.

La respuesta previa que establecemos es: El Sistema de Aprendizaje Basado en la Investigación facilita la formación con calidad y pertinencia profesional del estudiante de la carrera de Optometría con un sentido social, cumpliendo así con la responsabilidad institucional de formar profesionales con las aptitudes y actitudes que la sociedad demanda en materia de salud visual.

\subsection{PROCESO DE A IMPLEMENTACIÓN}

El proceso de enseñanza aprendizaje establecido en el Modelo Educativo del Instituto Politécnico Nacional, así como en el Modelo Educativo en Ciencias de la Salud propuesto por el Centro Interdisciplinario de Ciencias de la Salud, del propio IPN, se materializan con el uso del Sistema de Aprendizaje Basado en la Investigación, desarrollado por los docentes del CICS UMA 


\subsection{EVALUACIÓN DE LOS RESULTADOS}

En términos generales se presenta el proceso de operación en el aula del SABI:

1. Elección de contenidos a aprender por parte del profesor, siguiendo el programa sintético de la unidad de aprendizaje.

2. Determinación de proyecto de investigación final aplicando el proceso de aprendizaje durante el desarrollo de la unidad de aprendizaje objeto de estudio.

3. Documentos que se refieren al área de conocimiento de los contenidos, ofrecidos por el profesor a los alumnos a través de un sistema de almacenamiento de archivos en internet, Dropbox, para que éstos realicen lecturas críticas que permitan la explicación y análisis de los contenidos.

4. Listado de fuentes de información para realizar investigaciones sobre publicaciones científicas, tales como: Scielo, Sciencedirect, Elsevier, Pubmed, Redalyc, Dialnet, Google Scholar, y páginas del Instituto Politécnico Nacional (www.ipn.mx/publicaciones/), de la Universidad Nacional Autónoma de México (www.Librosoa.unam.mx), entre otros.

5. Envío al profesor, mediante correo electrónico, de las lecturas críticas elaboradas por los alumnos.

6. Registro y guarda de lecturas críticas en carpetas de evidencias individuales.

7. En el aula, introducción al tema de los contenidos por parte del profesor.

8. Integración de equipos de trabajo y designación de líderes para cada equipo.

9. Discusión y conclusiones por equipo de cada contenido, tomando como referencia las lecturas críticas que los alumnos tienen a la mano ya sea en su laptop o en algún otro medio electrónico: Tablet o celular. Cuando se cuenta con internet se puede utilizar el blog del grupo para bajar la información complementaria que previamente han subido los alumnos.

10. Presentación por parte de un equipo, seleccionado al azar, de sus conclusiones.

11. Discusión y aportaciones grupales, con lo que se llega a una propuesta grupal.

12. La propuesta grupal, además de ser enviada por el líder del grupo al profesor, se sube al blog del grupo para ser difundida y utilizada por cualquiera alumno que sea parte del blog.

13. Las lecturas críticas y las conclusiones se van integrando en las carpetas de investigación de cada equipo.

14. Durante el curso se va desarrollando el protocolo de investigación, alumno-profesor.

15. Una vez concluida la etapa de lecturas críticas, análisis y conclusiones grupales de todos los contenidos de la unidad de aprendizaje, se lleva a cabo la selección y ordenamiento de las lecturas críticas para poder construir el marco teórico conceptual del trabajo de investigación.

16. Realización del trabajo de campo, análisis de resultados, conclusiones y presentación de las propuestas, que emanan del proyecto de investigacióny que deben ser difundidas en la comunidad o enviadas a los grupos sociales que participaron en el trabajo de campo.

17. Publicación del producto de aprendizaje a través del proyecto de investigación. La publicación deberá hacerse por los medios que determinen los alumnos, sin embargo, se les induce a que lo hagan mediante las distintas propuestas que se manejan en internet.

Estos dos últimos puntos tienen como objetivo fundamental aportar a la sociedad conocimientos que apoyen la transformación de la realidad para elevar su nivel de bienestar, y en algunos casos como estrategia para el empoderamiento social de las acciones que la sociedad puede llevar a cabo para mejorar sus condiciones de salud. 
Cabe reiterar que nuestras propuestas no solo van en el sentido de prevenir enfermedades, se considera muy importante promover la salud, mediante la transformación de los determinantes sociales y el mejoramiento de su medioambiente.

De la aplicación del proceso del SABI a la Unidad de Aprendizaje Salud Pública con la generación 51 de la carrera de Optometría ${ }^{4}$, operada del 25 de febrero al 04 de marzo de 2019. Total, de alumnos 50.

Número de contenidos de la Unidad de Aprendizaje de Salud Pública: 25.

Total, de lecturas críticas: 1,250 .

Se revisaron que se hubiese cubierto la totalidad por cada alumno y de éstas se analizaron 10 lecturas de cada alumno. El control se realizó mediante carpetas electrónicas de cada alumno.

Temas específicos:

Dinámica poblacional de: México, Reino Unido, Suiza y Alemania, en 2010.

Brotes epidemiológicos:

Fiebre de tsutsugamushi en Vietnam, en el 2012.

Brote de leishmaniasis en Madrid, España, entre 2010 y 2011.

Pandemia de influenza AH1N1 en el año 2009.

Investigación: Tema. Síndrome del computador en jóvenes universitarios.

Como referencia de la aplicación del SABI, se presentan algunas evidencias del resultado de la formación de profesionales en el área de Optometría: lecturas críticas, dinámica poblacional, brotes epidemiológicos, carátulas de algunos proyectos de investigación sobre el síndrome del computador y ligas para la visualización de videos que reflejan el conocimiento adquirido y su disponibilidad para la sociedad, que se muestran en las imágenes de la 1 a la 6.

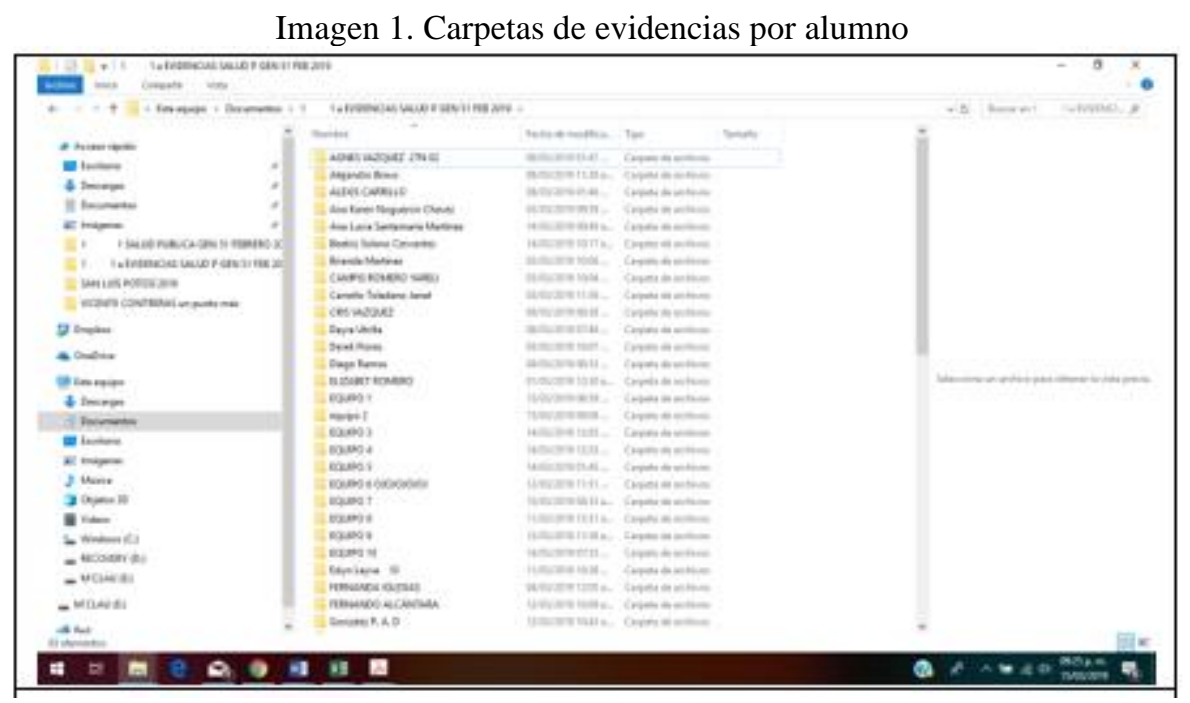


Imagen 2. Lectura crítica

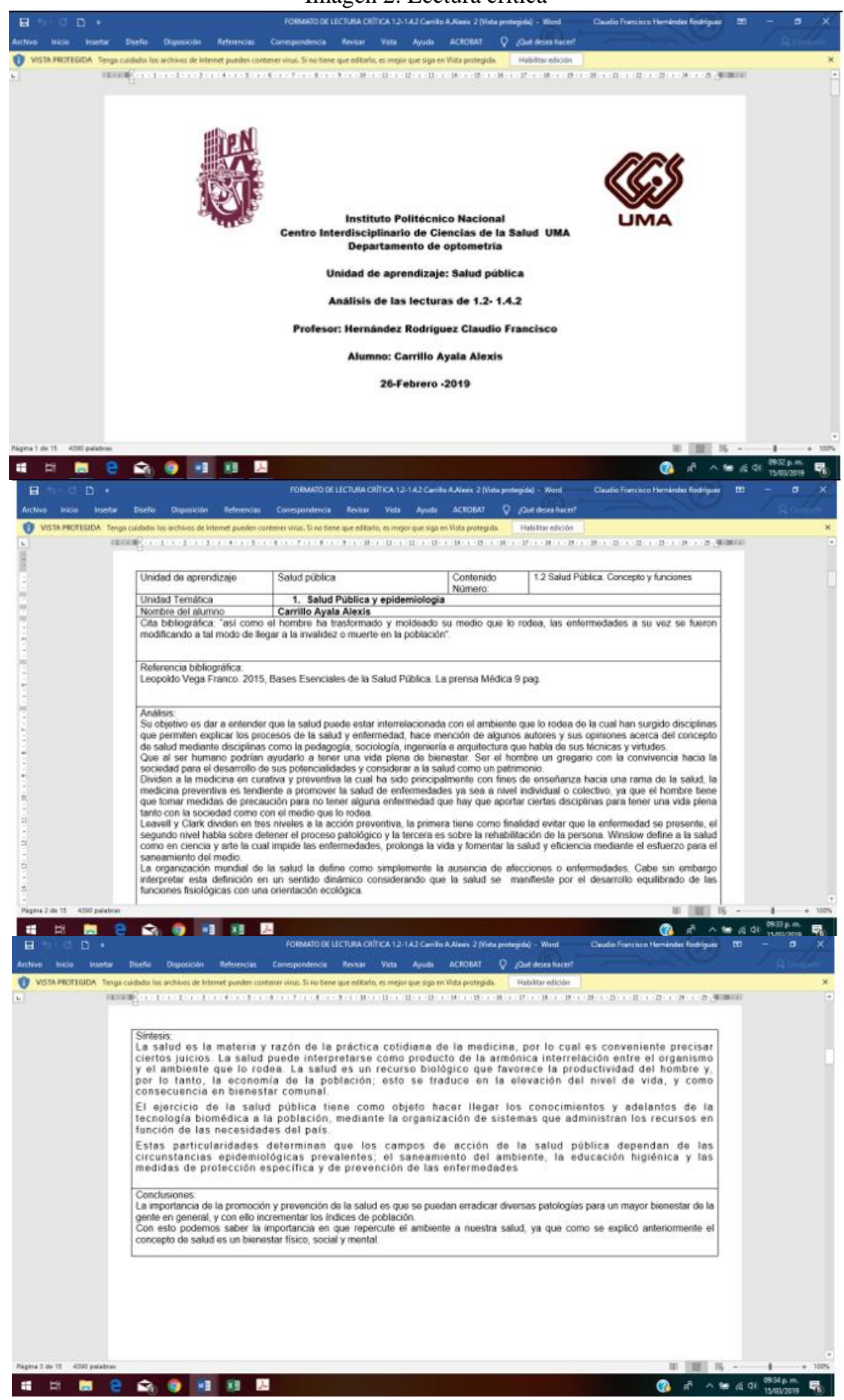


Imagen 3. Dinámica poblacional

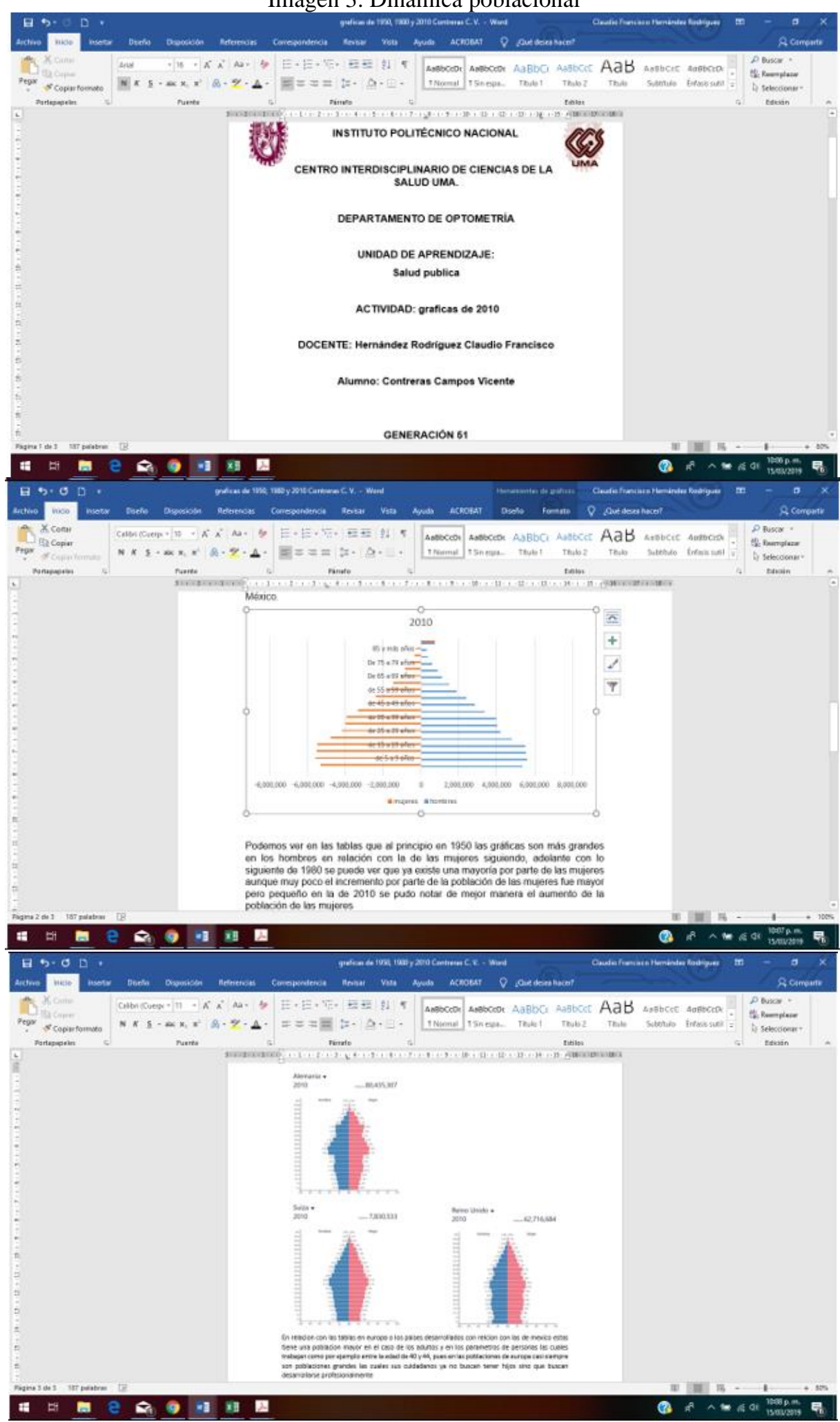


Imagen 4. Brotes epidemiológicos.

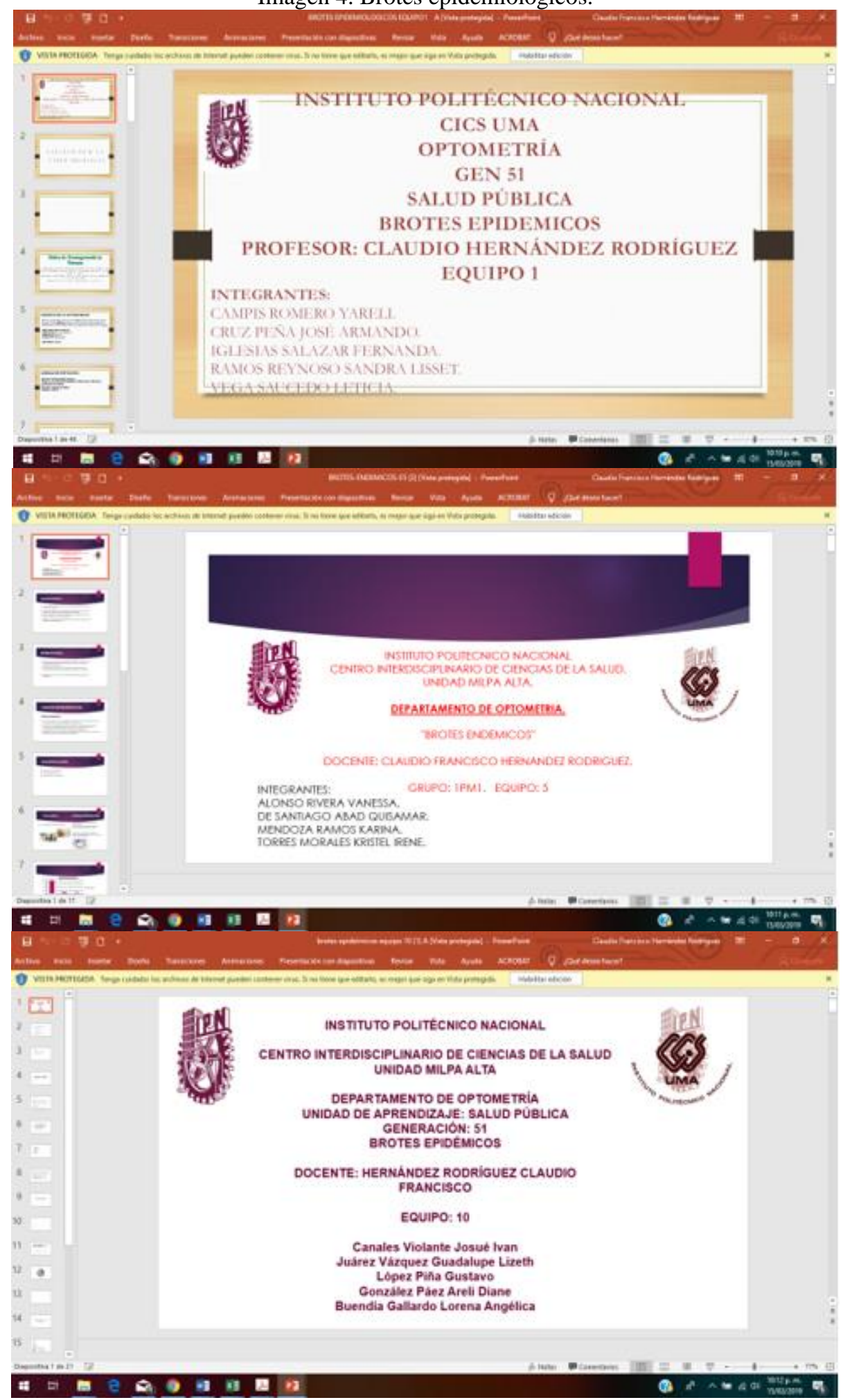


Imagen 5. Investigación sobre el síndrome del computador.

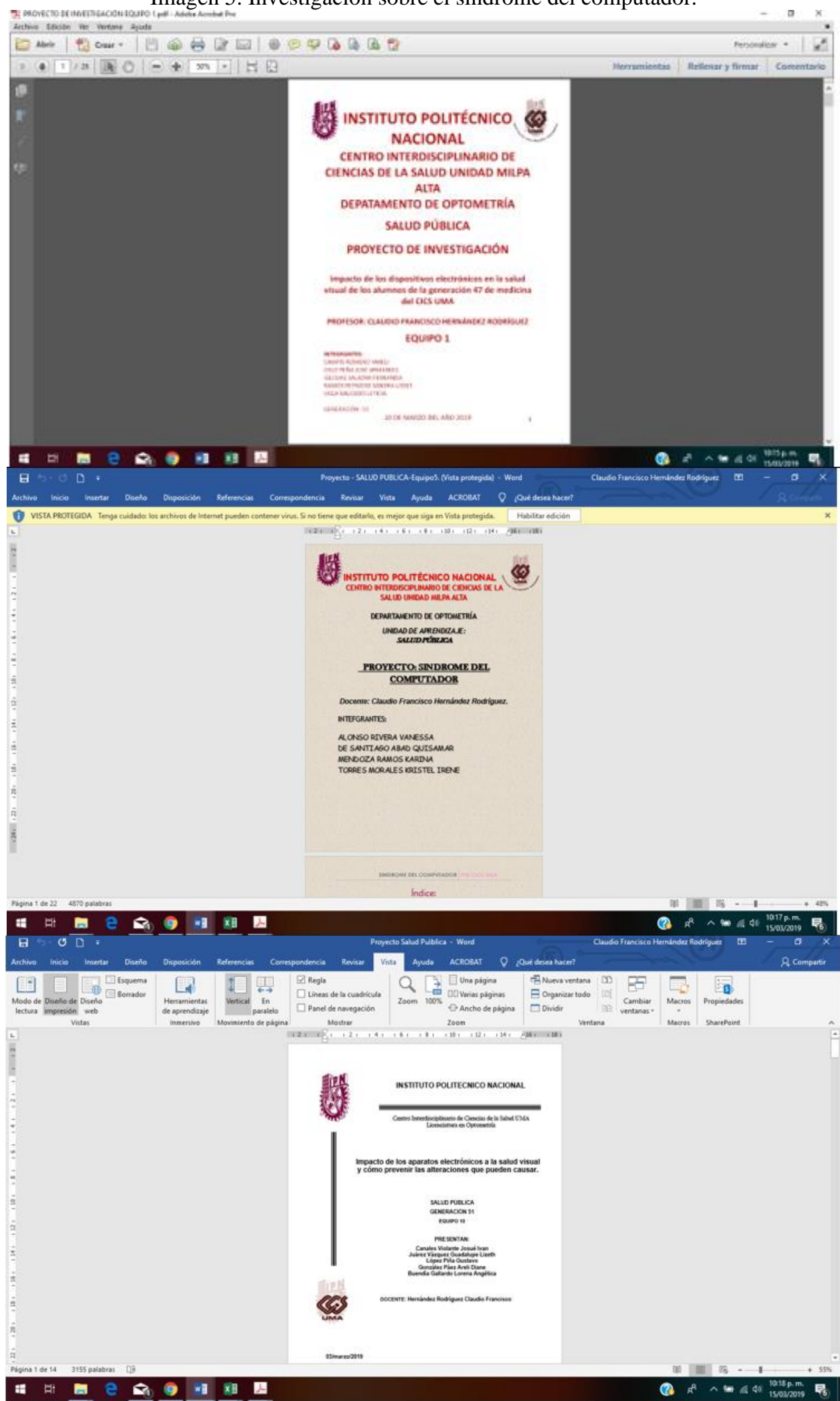


Videos:

Los videos que se produjeron a partir del proyecto de investigación, y que tienen como objetivo fundamental el empoderamiento social del conocimiento, para el cuidado de la función visual. Se puede tener acceso en:

https://youtu.be/TxesyvObvBg

https://www.youtube.com/watch?v=Jz_iwaRct8I\&feature=youtu.be https://www.youtube.com/watch?v=fY4VJImul3U\&feature=youtu.be https://www.youtube.com/watch?v=Lc5_c6ldU1M\&feature=youtu.be https://www.youtube.com/watch?v=-hQTNgFPQeA\&feature=youtu.be https://drive.google.com/file/d/1h1gGNebOyp8s38Wy9cNsr7JVK_zwPS9x/view https://drive.google.com/file/d/1AaSWPKlsZ9RV8eDFid6zHqqZdMBPL7LJ/view https://drive.google.com/file/d/1Jf03G9Z1FX3cQhZgwfQpxOYdLiQJwKmz/view?usp=sharing https://www.powtoon.com/online-presentation/bCwbnxdZg82/?mode=movie

Cabe señalar que todos los videos han quedado en sus carpetas de evidencias por equipo.

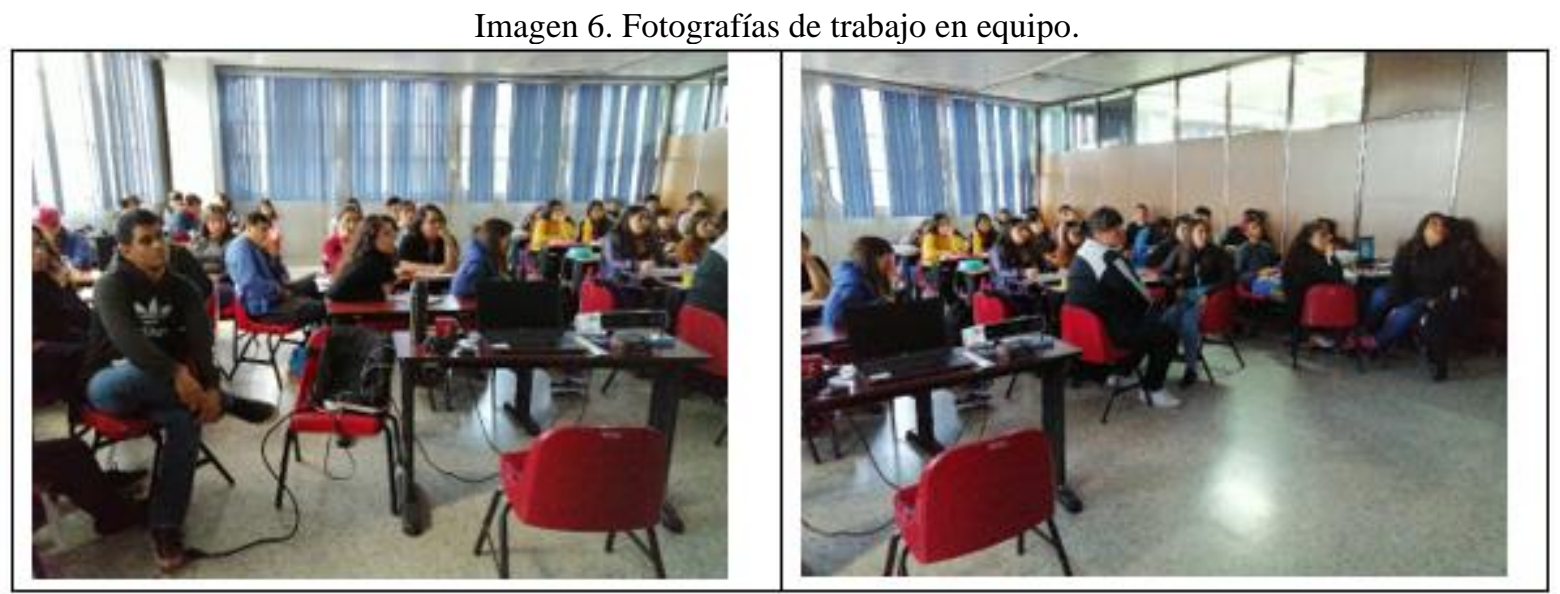

\section{CONCLUSIONES}

El Sistema de Aprendizaje Basado en la Investigación y el uso de las tecnologías de información y comunicación no solo favorecen la reconstrucción de nuevos conocimientos a partir de los conocimientos previos, sino que ayudan al alumno a mejorar sus habilidades cognitivas durante el propio proceso; así como a empezar a familiarizarse con las técnicas de investigación científica para hacer de ello un estilo de ejercicio profesional.

Además de lo anterior, los alumnos van tomando conciencia e inician, desde estudiantes, a cumplir con su función social dando respuesta a las necesidades de salud de la población utilizando los conocimientos adquiridos, en este caso, en el área de la optometría, para ser agentes activos en el proceso de empoderamiento social de los conocimientos y programas de salud visual.

La difusión por medio de los medios de comunicación e información, entre ellos YouTube y blog, de los conocimientos adquiridos sobre las medidas de prevención de daños por el uso desmedido de las 
herramientas de comunicación: teléfono celular, laptop, pantallas de TV, tables, entre otros, pueden ayudar a la población joven a cuidar su sistema visual, tomando en cuenta las medidas de prevención que los alumnos de la generación 51 de la carrera de Optometría difunden y explican, en base a los resultados de la investigación que realizaron como herramienta de su aprendizaje, es la evidencia del cumplimiento del CICS UMA de su compromiso social en el ejercicio de su función sustantiva: formar profesionales en el área de la salud y difundir el conocimiento en beneficio de la sociedad.

\section{PERSPECTIVAS}

Se está trabajando para operar la Unidad de Aprendizaje Salud Pública utilizando Classroom, con lo que se podrá cumplir con tres Políticas del CICS UMA: 1) Integración Práctica-Teoría-Práctica; 2) Desarrollo de la Comunidad y Compromiso Social y 3) Servicio Social Continuo, aplicando el SABI y teniendo como objetivo final continuar con la generación de conocimientos para la formación profesional de los alumnos y al mismo tiempo generar alternativas para el empoderamiento social de programas de prevención de enfermedades y promoción de la salud visual.

https://classroom.google.com/u/0/c/Mzl4NTMxOTMyMjda?hl=es

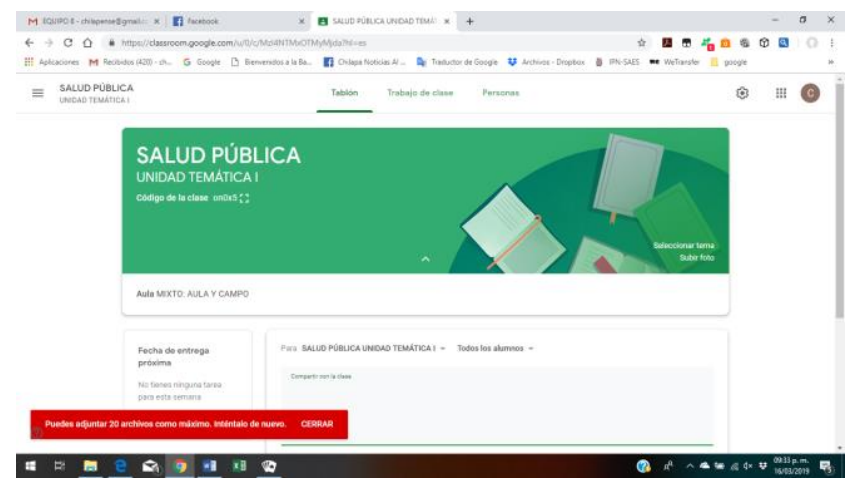




\section{BIBLIOGRAFÍA}

1 Un nuevo modelo educativo para el IPN. (2003). Instituto Politécnico Nacional. Dirección de Publicaciones. Primera edición: (2004). México, D. F. ISBN 970-36-0077-8

2 Documento CICS. Centro Interdisciplinario de Ciencias de la Salud. Subdirección Académica. Primera edición electrónica. (1979). México, D. F.

3 Sistema de Aprendizaje Basado en la Investigación. Hernández R. C. F \& Pérez M. B. E. (2015). Ex libris. México, D. F.

4 Programa Sintético: Unidad de Aprendizaje Salud Pública. (2014). Departamento de Optometría. Centro Interdisciplinario de Ciencias de la Salud, Unidad Milpa Alta. Programa México, D. F. 